\title{
Estimation of wall effects on floating cylinders
}

\author{
R. Porter and D.V. Evans \\ (richard.porter@bris.ac.uk, d.v.evans@bris.ac.uk) \\ University of Bristol, University Walk, Bristol, BS8 1TW, UK.
}

\section{Introduction}

Under the assumptions of classical linearised water wave theory, the time-harmonic twodimensional motion of a rigid body floating in the surface of a fluid may be characterised by various coefficients which express components of the hydrodynamic forces acting on that body.

For a single body in isolation these hydrodynamic coefficients are relatively simple to calculate. For bodies placed next to a vertical wall, the corresponding calculations are often much more complicated. Moreover, it is wellknown that the behaviour of a floating body in waves is radically affected by the proximity of a rigid boundary such as a harbour wall, and this effect is manifested in major changes to the hydrodynamic coefficients. This is due to the near-resonant excitation, close to certain frequencies, of waves trapped between the cylinder and the wall. Examples of the results obtained for bodies oscillating next to walls are given in Wang \& Wahab (1971) and Yeung \& Seah (2007) where these resonances are identified with large rapid variations in hydrodynamic coefficients.

In this paper we use the well-known widespacing approximation (see Martin (2006) for example) to develop approximations to the hydrodynamic coefficients for a body next to a wall solely in terms of the results for the forced motion the same body in the absence of a wall.

Exact results are compared with the widespacing approximations for semi-immersed circular cylinders and cylinders of rectangular cross-section and show excellent agreement.

\section{Formulation}

A two-dimensional cylinder is taken to be floating in the surface of a fluid of density $\rho$ and infinite depth. Cartesian coordinates are chosen with the origin in the mean free surface and $y$ pointing vertically downwards.

We consider the time-harmonic smallamplitude forced sway, heave or roll $(j=1,2$ or 3 respectively) motion of the cylinder centred at the origin in the presence of a rigid wall at $x=-b, y>0$ on which a Neumann condition is imposed on the potential, $\phi_{j}^{w}(x, y)$. (The superscript $w$ identifies quantities associated with the wall). Linearised water wave theory is used, in which potentials $\psi$ (say) satisfy $\nabla^{2} \psi=0$ in the fluid, $\psi \rightarrow 0$ as $y \rightarrow \infty$ and $\partial \psi / \partial y+K \psi=0$ on $y=0$ where $K=\omega^{2} / g, \omega$ being the angular frequency and $g$ gravitational acceleration.

The radiation potentials $\phi_{j}^{w}$ also satisfy

$$
\frac{\partial \phi_{j}^{w}}{\partial n}=n_{j}, \quad(x, y) \in S_{B} \quad(j=1,2,3)
$$

where $S_{B}$ is the wetted section of the floating body. In the case of sway and heave, $n_{j}$ are the direction cosines in the $x(j=1)$ and $y$ $(j=2)$ directions of the unit normal directed into the cylinder from the fluid. In the case of roll $(j=3)$ we have $n_{3}=x n_{2}+(y-c) n_{1}$ where $(0, c)$ is the point of roll.

As $x \rightarrow \infty$,

$$
\phi_{j}^{w}(x, y) \sim A_{j}^{w} \mathrm{e}^{\mathrm{i} K x-K y}, \quad(j=1,2,3)
$$

in which the far-field radiated wave amplitude $A_{j}^{w}$ is to be determined. Other quantities of interest are the added inertia and 
radiation damping coefficients $a_{j k}^{w}$ and $b_{j k}^{w}$ which define the real and imaginary parts of the complex time-independent restoring force matrix $f_{j k}^{w}$ representing the hydrodynamic force in the component $k$ due to a forcing in mode $j$, defined by

$$
f_{j k}^{w} \equiv-b_{j k}^{w}+\mathrm{i} \omega a_{j k}^{w}=\mathrm{i} \rho \omega \int_{S_{B}} \phi_{j}^{w} n_{k} \mathrm{~d} s .
$$

It is easy to show that $f_{j k}^{w}=f_{k j}^{w}$ and also that

$$
b_{j k}^{w}=\frac{1}{2} \rho \omega A_{j}^{w} \bar{A}_{k}^{w}, \quad(j, k=1,2,3)
$$

which are all real and it follows that $b_{j k}^{w} b_{k j}^{w}=$ $b_{j j}^{w} b_{k k}^{w},(j, k=1,2,3)$.

One final quantity of interest is the exciting force on a fixed cylinder in direction $j$ (see Mei (1983, p.302)) induced by waves of amplitude $A$ incident from $x=+\infty$ which is

$$
f_{S, j}^{w}=\mathrm{i} \rho \omega \int_{S_{B}} \phi_{S}^{w} n_{j} d s
$$

where $\phi_{S}^{w}$ is the scattered potential in the presence of a wall with $\partial \phi_{S}^{w} / \partial n=0$ on $S_{B}$. Here, it can be shown that

$$
f_{S, j}^{w}=\rho g A A_{j}^{w}, \quad(j=1,2,3) .
$$

\section{Wide-spacing approximation}

The overall effect of the wall will be equivalent to a radiated wave field travelling away from the cylinder in the absence of the wall, together with an incident wave of unknown amplitude $\left(D_{j}\right.$, say) from the left being scattered by the fixed cylinder (assuming the wall is far enough away from the cylinder). Thus

$$
\phi_{j}^{w}=\phi_{j}+D_{j} \phi_{S}, \quad(j=1,2,3),
$$

where $\phi_{j}$ is the radiation potential for a cylinder making sway, heave or roll $(j=1,2$ or 3$)$ motions at the origin but in the absence of the wall and $\phi_{S}$ is the scattered potential due to a wave incident from $x=-\infty$ on the cylinder held fixed at the origin, again in the absence of the wall.
We have the far-field expressions for each of the potentials in (7) given by

$$
\phi_{j} \sim\left\{\begin{array}{l}
(-1)^{j} A_{j} \mathrm{e}^{-\mathrm{i} K x-K y}, \quad x \rightarrow-\infty \\
A_{j} \mathrm{e}^{\mathrm{i} K x-K y}, \quad x \rightarrow+\infty
\end{array}\right.
$$

where $A_{j}$ are the far-field radiated wave amplitudes (left-right symmetry of the cylinder is assumed for simplicity) and

$\phi_{S} \sim\left\{\begin{array}{l}\frac{g A}{\omega}\left(\mathrm{e}^{\mathrm{i} K x}+R \mathrm{e}^{-\mathrm{i} K x}\right) \mathrm{e}^{-K y}, \quad x \rightarrow-\infty \\ \frac{g A}{\omega} T \mathrm{e}^{\mathrm{i} K x-K y}, \quad x \rightarrow+\infty\end{array}\right.$

where $R$ and $T$ are the reflection and transmission coefficients for the fixed cylinder, in the absence of the wall, due to an incident wave of amplitude $A$.

It is assumed that $A_{j}, R$ and $T$ are all known, in addition to $a_{j k}, b_{j k}$, the added inertia and radiation damping coefficients in mode $k$ due to forced motion in mode $j$, defined as in (3) but without the $w$ superscript.

It follows from (7) that for large positive $x$

$$
\phi_{j}^{w} \sim\left(A_{j}+(g A / \omega) D_{j} T\right) \mathrm{e}^{\mathrm{i} K x-K y}
$$

and for large negative $x$

$$
\begin{aligned}
\phi_{j}^{w} \sim\left((-1)^{j} A_{j}+(\right. & \left.g A / \omega) D_{j} R\right) \mathrm{e}^{-\mathrm{i} K x-K y} \\
& +(g A / \omega) D_{j} \mathrm{e}^{\mathrm{i} K x-K y} .
\end{aligned}
$$

These asymptotic forms are now assumed to hold near the wall along $x=-b, y>0$ where a Neumann condition is now imposed on the potential $\phi_{j}^{w}$. It follows that

$$
(-1)^{j} A_{j}+(g A / \omega) D_{j} R=(g A / \omega) D_{j} \mathrm{e}^{-\mathrm{i} \lambda}
$$

with $\lambda=2 K b$ whence

$$
D_{j}=(\omega / g A)(-1)^{j} A_{j} /\left(\mathrm{e}^{-\mathrm{i} \lambda}-R\right) .
$$

Substituting (13) into (10) and comparing with (2) gives

$$
A_{j}^{w}=\delta_{j} A_{j}
$$

where 


$$
\delta_{j}=\left(\frac{R-(-1)^{j} T-\mathrm{e}^{-\mathrm{i} \lambda}}{R-\mathrm{e}^{-\mathrm{i} \lambda}}\right)
$$

Note that this implies $\delta_{1}=\delta_{3}$. According to the decomposition made in (7), the restoring force matrix, from (3), is approximated under the wide-spacing approximation by

$$
f_{j k}^{w} \equiv-b_{j k}^{w}+\mathrm{i} \omega a_{j k}^{w}=f_{j k}+D_{j} f_{S, k}
$$

where $f_{S, k}=\rho g(-1)^{k} A A_{k}$.

Notice that (16) only holds provided $j+k$ is even since if $j+k$ is odd, then the symmetry of the cylinder implies that the term $f_{j k}$ is identically zero (for example, a heave motion induces neither sway force nor roll moment on a symmetric cylinder) and (16) is replaced with

$$
f_{j k}^{w}=D_{j} f_{S, k} .
$$

Essentially (16) and (17) define, via (13), the wide-spacing approximations to the added inertia and radiation damping for a cylinder in the presence of a wall in terms of the solution to the wave radiation and scattering by a cylinder in isolation. Additionally (14), via (15), defines the far-field radiated wave amplitudes.

However, we can make further progress by manipulating the equations (16) and (17) that define $a_{j k}^{w}, b_{j k}^{w}$ using relations such as

$$
b_{j k}=\frac{1}{2} \rho \omega\left(1+(-1)^{j+k}\right) A_{j} \bar{A}_{k}
$$

(clearly zero if $j+k$ is odd) and the Newman/Bessho relations (see Mei (1983), p.328)

$$
R+(-1)^{j} T=-A_{j} / \bar{A}_{j}=-\mathrm{e}^{2 \mathrm{i} \theta_{j}},
$$

where $\theta_{j}$ is the phase of the far-field radiated amplitude in the $j$ th mode (note $\theta_{1}=\theta_{3}$ ).

We omit the details here and summarise below the simplified forms of the approximations to $a_{j k}^{w}, b_{j k}^{w}$.

For $j+k$ odd, we obtain,

$$
b_{j k}^{w}=\frac{2\left(b_{j j} b_{k k}\right)^{\frac{1}{2}} \cos \mu_{j} \cos \mu_{k}}{\left|\mathrm{e}^{-\mathrm{i} \lambda}-R\right|^{2}}
$$

and

$$
\omega a_{j k}^{w}=\frac{-\left(b_{j j} b_{k k}\right)^{\frac{1}{2}} \sin \left(\mu_{j}+\mu_{k}\right)}{\left|\mathrm{e}^{-\mathrm{i} \lambda}-R\right|^{2}}
$$

where

$$
\mu_{j}=\theta_{j}+K b .
$$

For $j+k$ even,

$$
b_{j k}^{w}=\frac{2 b_{j k} \cos ^{2} \mu_{l}}{\left|\mathrm{e}^{-\mathrm{i} \lambda}-R\right|^{2}}
$$

where $l$ is either $k+1$ or $k-1$ provided that number falls in the set $\{1,2,3\}$ whilst

$$
a_{j k}^{w}=a_{j k}-\left(\beta_{k} / \omega\right) b_{j k}
$$

where

$$
\beta_{k}=\frac{\frac{1}{2}(-1)^{k} \sin 2\left(\theta_{l}-\theta_{2}\right)-\sin 2 \mu_{k}}{\left|\mathrm{e}^{-\mathrm{i} \lambda}-R\right|^{2}}
$$

and here $l$ is either 1 or 3 .

\section{Results}

We show, in figures 1 and 2, two sets of results for the non-dimensional ${ }^{1}$ added inertia and radiation damping coefficients, varying with non-dimensional frequency. In each set of figures, the solid lines correspond to exact calculations including the wall and the points are calculated using the wide-spacing approximation (20), (21), (23), (24).

In figure 1 , results are shown for a semiimmersed circular cylinder of radius $a$, whose centre is a distance $b=2 a$ from the wall. In this example, there is no roll component. As expected, the wide-spacing approximation performs better as $K a$ increases, but still does remarkably well as $K a \rightarrow 0$.

In figure 2 , results are shown for a floating rectangular cylinder of width $2 a$, draught $d=2 a$ centred a distance $b=4 a$ from the wall. The fluid is now of finite depth $h$

\footnotetext{
${ }^{1} \mu_{j k}^{w}=a_{j k}^{w} / M, \nu_{j k}^{w}=b_{j k}^{w} /(\omega M)$ for $j, k=1,2$ where $M$ is the mass of the cylinder, determined by Archimedes' principle. Also, $\mu_{33}^{w}=a_{33}^{w} / I, \nu_{33}^{w}=$ $b_{33}^{w} /(\omega I)$ and $\mu_{j 3}^{w}=a_{j 3}^{w} / \sqrt{M I}, \nu_{j 3}^{w}=b_{j 3}^{w} /(\omega \sqrt{M I})$, $j=1,2$ where $I$ is a moment of inertia about $(0, c)$
} 

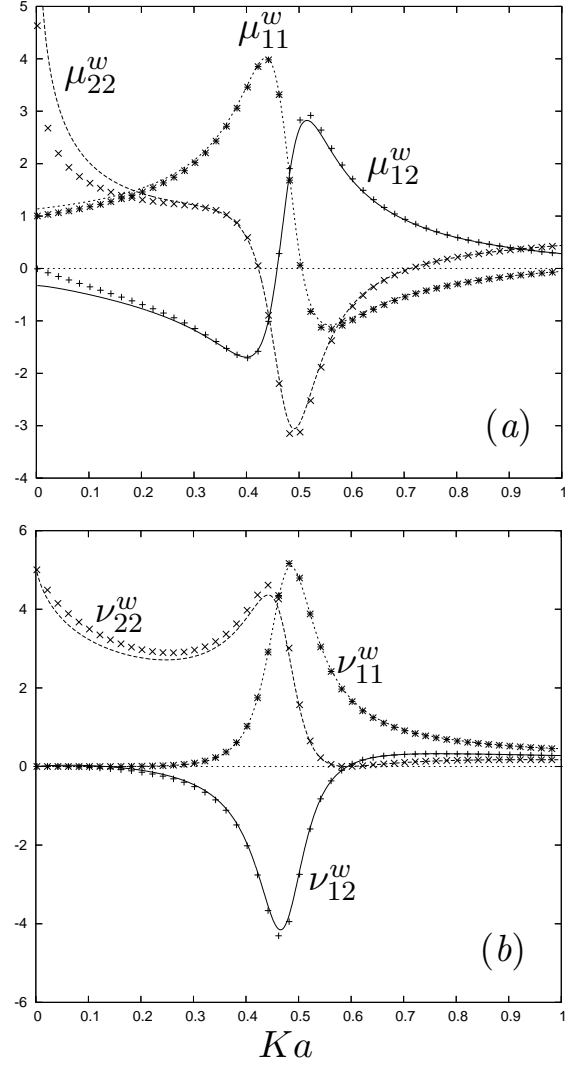

Figure 1: Variation of non-dimensional $(a)$ added mass and (b) radiation damping coefficients for a circular cylinder with $K a$.

$(=5 d)$, the wavenumber $k$ determined from $K=k \tanh k h$. Again the wide-spacing results, compared to exact calculations are in excellent agreement across the range of frequencies.

\section{References}

1. Martin, P.A., 2006. Multiple Scattering: Interaction of Time-Harmonic Waves with $N$ Obstacles. Cambridge University Press.

2. Mei, C.C., 1983, The Applied Dynamics of Ocean Surface Waves. WileyInterscience.

3. Yeung, R.W. \& Seah, R.K.M., 2007, On Helmholtz and higher-order resonance of twin floating bodies. J. Eng. Math., 58, 251-265.

4. Wang, S. \& Wahab, R., 1971, Heaving oscillations of twin cylinders in a free surface. J. Ship Res., 15(1), 33-48.
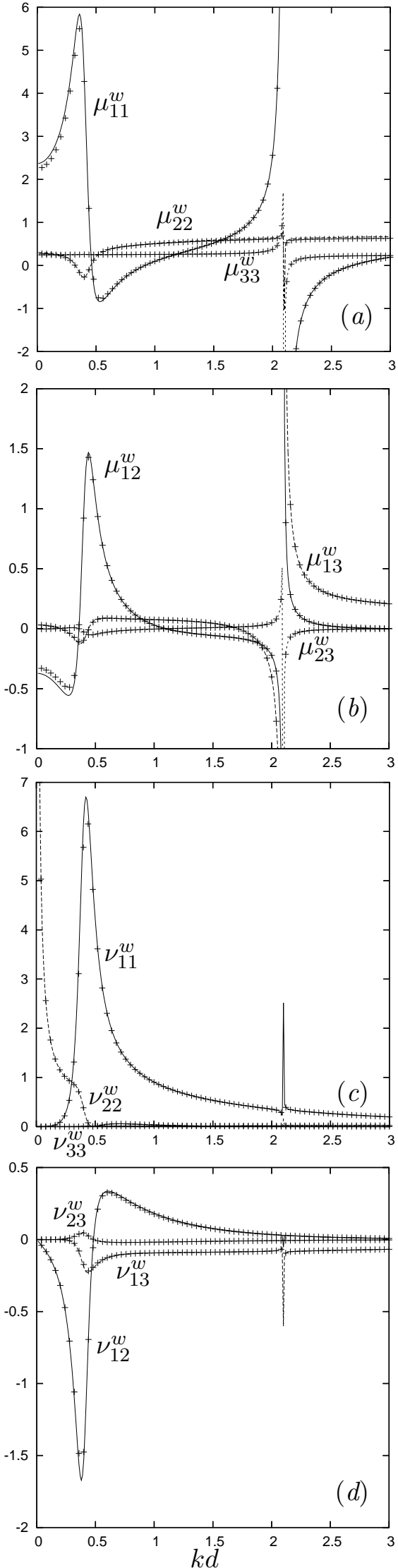

Figure 2: Variation of non-dimensional $(a, b)$ added inertia and $(c, d)$ radiation damping coefficients for a rectangular cylinder with $k d$. 